\title{
ON THE EMERGENCE OF PATTERNS OF ORDER
}

\author{
J. M. BURGERS
}

1. Introduction. The lecture which I have been asked to give is meant to honor a scientist whose work has encompassed a wide domain of knowledge. I have thought that a theme for tonight could be found in a review of some aspects of regularity and order as they appear in systems considered in mathematical physics. As this presents a very wide subject, I can do no more than touch upon a few examples.

Forms of order constitute the most basic fact which faces us in the phenomena of nature, and to become aware of a pattern of regularity has always been a striking experience. It has often evoked an attitude of respect for the forces of nature, and it has never ceased to tempt the mind to speculate what causes there may be behind the regularity. Even the question: Has this order a specific meaning? Does it point to something? has often turned up. Such a question, however, reaches beyond the realm of pure science, since any arrangement can be considered as representing some form of order when we take the mathematical point of view, and no form of order can then be said to be more important or to have more meaning than any other form.

It may be appropriate to review briefly certain cases of appearance of order and to focus attention upon the features from which they derive. Although this will not lead to new results, a kind of panorama outlook can have an attraction for itself. It is interesting to go over some of the steps which mark their explanation and to collect the essential points as far as possible into a single picture.

I will try to achieve this by describing a few examples of patterns in spatial arrangement. The first example will refer to crystal structure; the second one to a case of fluid motion; finally I will consider a mathematical equation describing an extremely simplified case of gas motion, under such circumstances that no pressure arises. For lack of time I leave aside phenomena of order in temporal sequences and shall not speak of periodic motions, etc.

When we start from the usual conceptions of Euclidean space in which, notwithstanding relativity and gravitation, most phenomena are still described, we are faced with its continuity and homogeneity. A primary requirement for the appearance of a pattern in such a

The thirty-second Josiah Willard Gibbs lecture delivered before the Annual Meeting of the Society in Philadelphia on Tuesday, January 20,1959; received by the editors February, 1959 (the unfortunate delay in publication is due to the fact that the manuscript was subsequently misplaced). 
space is something that supplies us with a basic length, a basic distance between similar features. Once such a distance is given, patterns can emerge through repetition.

From where do discrete lengths arise in our description of the structure of matter? One answer is that many phenomena of nature can be described by means of linear differential equations with which systems of eigenfunctions and eigenvalues are associated. It is this feature which can introduce a peculiar discreteness within continuous Euclidean space. Still, this answer is not a final one. The formulation of an eigenvalue problem requires the existence of boundary conditions. If these conditions only refer to the origin and to infinity, they do not themselves introduce any scale. There remains the feature that the differential equations of physics contain certain constants of nature, which have definite values. Thus in the theory of the structure of atoms we encounter the mass of the electron $m$, the unit electric charge $e$, and Planck's constant $h$. It is from these constants that fundamental dimensions and distances arise. It would be outside the scope of this lecture to speculate about the background of these constants of nature and about their relation to other constants, as the velocity of light and the constant of gravitation. We shall simply take them for granted.

It must be observed that in mathematical physics we often also have to do with differential equations supplemented by conditions referring to boundaries at finite distances within the field. In such cases these boundary conditions introduce definite dimensions. From the point of view of the differential equation they may appear to be "accidental," as they depend upon circumstances more or less outside the mechanism described by the equation itself.

I shall consider an example of each one of the two cases mentioned here. From boundary conditions we are led towards initial conditions, and the third example will refer to a case where the state of the system at time $t=0$ is the important datum.

2. Crystal structure. The most conspicuous example of the first class of systems is the order exhibited by crystals. This order is the outcome of the atomic lattice structure. When we assume atomic nuclei and electrons as given quantities, the following principles enter into the structure of these lattices.

From the three quantities $m, e, h$ mentioned before, a primary unit of length can be derived, the so-called "first Bohr radius" $a=h^{2} / 4 \pi^{2} m e^{2}$. This quantity fixes the scale for the eigenfunctions of the Schrödinger equation for the simplest atom, a hydrogen atom in unlimited space.

In the structure of atoms with more electrons two further principles 
are involved, viz. that the charge of the atomic nucleus is an integer multiple of the unit charge; and Pauli's principle, according to which all electrons of an atom must be in different quantum states. The rules of quantum theory then completely determine the dimensions of the electronic system. On the basis of the same principles a theory has been developed of the forces between two or more atoms. The equilibrium positions between repulsion and attraction determine a set of "secondary distances," characteristic for atomic combinations.

The final feature is the possibility of an unlimited repetition of a basic pattern of arrangement. In this repetition the distances and angular relations occurring in an elementary cell of the structure are reproduced with great precision, often ten million times and more in each coordinate direction. We find here a transmission of order through space, and it is in this way that crystals of macroscopic dimensions arise.

I will not go into the details of these arrangements and bother you with an enumeration of space lattices or symmetry classes. Rather I will follow a sidetrack. I remind you that a physicist, once he has understood the principles, does not content himself with a contemplation of forms of order, however beautiful, but is inclined to look for infringements upon the ideal rules. The more elaborate the structure, the more possibilities there are for infringements.

In the subject of crystal structure there are two aspects which call for attention in this connection.

The first point is that it follows from thermodynamical theory that the order exhibited in a crystal is a by-product of a tendency towards variety. This is best elucidated by the following consideration of the conditions for crystallization.

Suppose that we have a large collection of atoms of suitable types within a closed volume, endowed with a given amount of energy. The energy will be present partly as kinetic energy, partly as potential energy dependent upon the attractions between the atoms. In such a system a large number of configurations are possible. These are different quantum states for the system, all belonging to the same total energy; they can be distinguished and counted according to the methods of quantum theory. In some configurations all atoms may have that type of irregular motion which we are accustomed to ascribe to the molecules of a gas. In other configurations some atoms may form a crystal lattice, while only the rest are in gaseous motion. There is a perpetual change from configuration to configuration, and what we observe as the "macroscopic state" of the system is actually an average result over an enormous number of alternating configurations.

One can calculate how many of these configurations, all of which 
have the same weight in the theory, are compatible, for instance, with the condition that $x$ per cent of the matter shall be condensed in the form of a crystal lattice. It has been found that the equilibrium state of the system ("state" again in the macroscopic sense) belongs to that value of $x$ for which the number of configurations has the largest value. In systems consisting of large numbers of atoms, such as we encounter in ordinary circumstances, the number of configurations belonging to this equilibrium state completely outweighs the number of configurations for any other state.

When the energy of the system is high, the largest number of configurations is obtained by having all atoms in the gaseous form. On the other hand, when the energy of the system falls below a certain limit, it is advantageous to bring together a number of atoms into a lattice structure, which greatly reduces the potential energy of these atoms. The energy released by this process becomes available as kinetic energy and this leads to a much greater number of possible configurations than can be realized if all the atoms were to remain in the gaseous form.

We conclude that in a physical system there can appear no more than a partial order, since the energy liberated by the ordering must leave room for variety in configurations in other aspects of the system. When the system is not closed but is in contact with surroundings, part of the variety of configurations can be realized in the surrounding matter. Thus the law that a crystal is an arrangement of minimum potential energy is a result of the rule that somewhere, either in the system itself, or in its surroundings, the largest possible number of configurations must be realized. In a certain sense we can say that the emergence of order is conditioned by the possibility of configurational variability elsewhere, by preference in surroundings which we are inclined to consider as less interesting than the system itself.

The most perfect order that can be attained in a system is to have it in a single quantum state which is prevented from alternating with other quantum states. In principle this requires that there are no other quantum states with the same energy. In many cases this can only be realized when the system is in the quantum state of lowest energy. Thus order in physical systems is mostly dependent upon the possibility of detracting energy from the system, for instance by radiating energy away into unbounded space. The circumstance that there is so much almost empty space around us, with an extremely low energy level, is of great importance in this connection.

3. Imperfections in crystal lattices. The second point to be con- 
sidered is that the order in a crystal lattice is never perfect. Already surfaces where crystals of different orientation meet, cannot have the regular lattice structure. But even within a single lattice there can be vacant sites, superfluous atoms in interstitial positions, or atoms of a wrong type; and also errors in the fitting together of lattice planes, which errors are called "dislocations." These imperfections usually concern only a small percentage of the lattice sites; nevertheless, they are of great importance for various physical properties of the crystallized material. ${ }^{1}$

Dislocations form a very interesting class of imperfections and bring with them a special geometry. They are characterized by a line, the "dislocation line," indicating the situation of the misfit; and by a characteristic vector, measuring the magnitude of the misfit. The characteristic vector (sometimes called Burgers vector) must be a vector occurring in the lattice structure of the crystal. When it is a unit vector of the elementary cell and thus a full period of the lattice, the dislocation is termed a perfect dislocation. Such a dislocation introduces no other misfit than what is found along the dislocation line, which plays the part of a singularity in the structure; all the rest of the lattice has its normal or "healthy" arrangement. The characteristic vector can also be another vector occurring in the elementary cell, less than a full period. Such a dislocation is called imperfect; in distinction of a perfect dislocation it is characterized by a surface (either plane or curved) over which the normal lattice structure is disturbed, for instance by passing into a mirror image as occurs in twinning; or by a deviation from the normal stacking order in lattices consisting of close packed sheets of identical spherical atoms. In this case the dislocation line is the contour of the disturbed area.

A perfect dislocation has a certain resemblance with a vortex line in hydrodynamics and cannot end within the lattice. Dislocation lines can form nodes where three or more branches meet; the sum of the characteristic vectors associated with the branches must then be zero provided a certain rule of signs is observed. When the characteristic vector is perpendicular to the direction of the dislocation line, the dislocation is of "edge" type; in this case a layer of atoms ends at the dislocation line, thus forming an edge; beyond this edge the neighboring layers on both sides must approach each other over a distance equal to the characteristic vector, in order to restore normal lattice arrangement. When the characteristic vector is tangential to the dislocation line, the dislocation is of "screw" type; the lattice planes then are fitted together as a helical surface winding around

${ }^{1}$ Reviews of the subject of lattice defects are given in $[1,2]$. For the theory of dislocations see $[3,4]$. 
the dislocation line. There can also be dislocation lines, or portions of dislocation lines, which are of intermediate (mixed) type.

Similar properties hold for imperfect dislocations, but they bring more complicated situations.

Dislocations originate during the growth of a crystal, and also in processes of deformation. Moreover, the presence of screw dislocations can make crystal growth more rapid. This is due to the circumstance that a crystal grows most easily when atoms can find a position along an edge of a terrace on a crystal face, and it is the particularity of a screw dislocation that it presents an edge which never disappears in the process. The growth determined by such a dislocation can lead to curious patterns on the crystal faces. ${ }^{2}$

An important property of dislocations is that, with the exception of some types of imperfect dislocations, they can migrate through the lattice. This involves, of course, that certain rows or planes of atoms in the lattice move relatively to each other.

There are two types of migrations. One type is "conservative migration," which does not involve the creation of new vacant lattice sites or the introduction of new atoms (such as either may come into the lattice by diffusion, or may previously have found a place in interstices between regular lattice positions). Conservative migration can occur in an ideal form when the lattice has no other imperfections. This type of migration plays a part in the explanation of plastic deformation of crystals under shearing stresses. Some forms of this process, in which the dislocation line is held fixed at certain points, can lead to the extension or to the multiplication of dislocation lines in a lattice. Conservative migration furthermore can play a part in the transformation of a lattice structure into one of a different type, for instance, into a symmetrical arrangement as occurs in twinning, or in the change from a lattice of cubic face-centered structure into one of hexagonal type. For each of the changes in structure which have been observed to occur, a particular mechanism must be found; this usually requires an elaborate comparison of various possibilities for migrations. For instance, to produce a change of lattice type in a crystal lattice consisting of a single species of atoms, one needs the presence of a screw dislocation together with an imperfect dislocation, which latter must be able to turn around the screw axis and follow the helical surface determined by the screw. More complicated combinations have been imagined for changes in crystals with more than one species of atoms. ${ }^{3}$

See $[5 ; 6]$.

${ }^{8}$ See W. T. Read, [3]; J. M. Burgers and W. G. Burgers, [4], pp. 178-180.

An example referring to a lattice containing two species of atoms is treated by M. L. Kronberg, [7]. 
Another type of migration, nonconservative migration, can occur when the lattice has superfluous atoms or vacancies; the migration may then bring about that superfluous atoms obtain proper lattice positions, or that the vacancies disappear. On the other hand, it is also possible that migration of the dislocation will create vacancies, or will produce superfluous atoms. ${ }^{4}$

Anyone who glances over the literature on this subject will be struck by the fact that here, out of the concept of structural "errors," a theory of new forms of order has developed. Add to this the fact that in certain varieties of crystals structural defects may repeat themselves in a more or less regular way. A spiral dislocation, for instance, can be the cause that a deviation in a part of a lattice plane is propagated along the screw surface and becomes a regularized feature of a crystal. ${ }^{5}$ Here again, structural deviations cease to be "errors" and become evidence for the presence of a superstructure in the crystal. Thus it is seen that there is an interplay between the concepts of "order" and "disorder." The human mind continually looks for descriptions and interpretations which reveal new forms of order.

4. Fluid motions resulting from instability. I will now pass to an example of the second class, in which we perceive a dominant influence of a boundary condition. We leave the domain of atomic physics and consider a case of fluid motion, treating the fluid as a continuum. The fundamental dimensions of nature, so evident in crystal lattices, now will appear only in disguised form, through the values of such macroscopic properties as density, viscosity and heat conduction (sometimes also in the effects of capillarity or other physical forces).

We take a case where a fluid finds itself in an unstable situation, so that a small disturbance can give rise to the appearance of a particular form of motion. An instance which has been investigated both experimentally and theoretically refers to the convection currents which can arise in a liquid heated from below. ${ }^{6} \mathrm{~A}$ layer of fluid

4 Nonconservative migration can occur only at temperatures where diffusion of atoms or of vacancies through the lattice can be of importance.

See: J. Bardeen and C. Herring, in W. Shockley $[1]$, p. 261 ; and $[8 ; 9 ; 10]$.

-See [6, p. 86, "Le polytypisme"].

- The original observations have been made by Henri Bénard, see $[11 ; 12]$.

For later experimental work see [13].

Mathematical investigations have been carried out by Lord Rayleigh, H. Jeffreys, and A. R. Low; see for instance [14]. Some newer calculations have been given by W. H. Reid and D. L. Harris, see [15].

A review of the older investigations, in connection with applications to astrophysical problems, is given in [16]. 
is supported by a horizontal surface which is heated, while the top layer of the liquid is in contact with a cold horizontal confining surface, or simply with cold air. The lower layers will obtain a smaller density than the upper layers in consequence of their higher temperature, and the situation is unstable. The instability can release itself through currents which transport hotter liquid from the bottom towards the top, at the same time bringing cooler liquid downward. The colder liquid becomes heated in its turn, while the liquid transported upward will lose heat by contact with the colder surroundings; thus the current system can persist. The peculiar feature observed is that under favorable circumstances the convection currents assume a pattern of polygonal cells of approximately equal dimensions, and sometimes of quite regular form.

The theoretical analysis of the motion assumes that the velocity of the liquid is small, so that linearized equations can be used. Account must be taken of the change in density due to variation of temperature, of heat conductivity in regulating the temperature field, and of viscosity in regulating the speed of flow. A linear partial differential equation is obtained for the vertical velocity of the liquid; the solution of this equation leads to an eigenvalue problem, in which the eigenvalues are connected with the temperature gradient leading to instability. ${ }^{7}$ One of the parameters in this equation is a quantity connected with the horizontal dimensions of the pattern of currents.

7 With the $z$-axis vertical and $w=$ vertical velocity of the liquid, the equation takes the form:

$$
\sigma^{2} \Delta w-(\kappa+\nu) \sigma \Delta^{2} w+\kappa \nu \Delta^{8} w=g \alpha \beta \Delta^{*} w,
$$

where $\sigma$ has been written for $\partial / \partial t ; \Delta$ for $\partial^{2} / \partial x^{2}+\partial^{2} / \partial y^{2}+\partial^{2} / \partial z^{2}$; and $\Delta^{*}$ for $\partial^{2} / \partial x^{2}$ $+\partial^{2} / \partial y^{2} ; g$ is the acceleration of gravity, $\beta$ the mean temperature gradient produced by the heating, $\alpha$ the thermal volume expansion coefficient of the liquid, $\nu$ the kinematic viscosity and $\boldsymbol{k}$ the thermometric heat conductivity. The same equation holds for the temperature perturbation $\theta$.

For limiting instability we replace $\sigma$ by 0 ; the equation reduces to: $\kappa \nu \Delta^{3} w=g \alpha \beta \Delta^{*} w$. We suppose that $w$ is of the form $W(x, y) \mathcal{Z}(z)$ and assume:

$$
\Delta^{*} \varpi=-\left(\lambda^{2} / h^{2}\right) W \text {. }
$$

Writing $\zeta=z / h$, where $h$ is the thickness of the layer, the equation for the function $\mathcal{Z}(\mathbf{z})$ becomes:

$$
\left(d^{2} / d \zeta^{2}-\lambda^{2}\right)^{3} Z=-p \lambda^{2} Z .
$$

Here $p=g \alpha \beta h^{4} / \kappa \nu$; this quantity can be taken as the eigenvalue parameter.

Various systems of boundary conditions for $\mathbf{Z}$ have been considered, depending upon the thermal and frictional conditions at the two surfaces limiting the liquid below and above. For each set of conditions and each value of $\lambda$ a series of eigenvalues is obtained for $p$; the problem investigated has been to find the value of $\lambda$ which makes the lowest eigenvalue a minimum. For instance, with two rigid conducting boundaries, $\lambda=3.17$ gives the minimum value of $p$ as 1709 . 
This dimension is not known beforehand. One has therefore calculated for which ratio of this dimension to the thickness of the layer the instability occurs with the smallest temperature gradient. Thus a principle is invoked which we may call "the principle of the most unstable solution," and it is assumed that the resulting pattern will depend upon the parameters governing this most unstable solution.

It must be observed that in this case the scale of the horizontal pattern (the parameter $\lambda$ ) is made dependent upon the thickness $h$ of the layer of liquid through the intermediary of a numerical quantity $(p)$, the value of which is dependent upon the structure of the differential equation and upon the nature of its boundary conditions. It must also be noted that even with a definite value of $\lambda$, the differential equation introduced for the amplitude $w$ of the vertical velocity at the instability limit, does not enforce a definite pattern. Boundary conditions for w have been deduced from the observational result that in most cases the liquid rises in the center of each cell, while a compensating downward movement takes place along the cell walls; from this it follows that the normal derivative (in the horizontal plane) of $\%$ must be zero at these walls. Even with this boundary condition the equation admits a variety of solutions. ${ }^{8}$ However, when we consider a few simple types of solutions, with boundaries in the form of a circle, a square and a hexagon, we readily guess that the hexagonal form with its obtuse corners will give the most probable solution. Some authors have suggested that nonlinear effects are of importance in this connection. ${ }^{9}$

${ }^{8}$ When $\lambda$ has been found, the horizontal pattern is governed by the equation:

$$
\frac{\partial^{2} W}{\partial x^{2}}+\frac{\partial^{2} W}{\partial y^{2}}=-\frac{\lambda^{2}}{h^{2}} W \text {. }
$$

Some simple solutions are:

(i) for a circle with radius $R$ :

$$
W=W_{0} J_{0}(\lambda r / h),
$$

provided: $J_{1}(\lambda R / h)=0$, from which $R / h=3.83 / \lambda$;

(ii) for a square with sides $2 a$ :

$$
\mathscr{W}=\frac{1}{2} W_{0}\left(\cos \frac{\pi x}{a}+\cos \frac{\pi y}{a}\right) \text {, }
$$

with $a / h=\pi / \lambda$;

(iii) for a hexagon with sides determined by

$$
\begin{gathered}
(x \pm a)(x+y \sqrt{ } 3 \pm 2 a)(x-y \sqrt{ } 3 \pm 2 a)=0: \\
W=\frac{1}{3} W_{0}\left\{\cos \frac{\pi}{a}\left(x+\frac{y}{\sqrt{ } 3}\right)+\cos \frac{\pi}{a}\left(x-\frac{y}{\sqrt{ } 3}\right)+\cos \frac{2 \pi y}{a \sqrt{ } 3}\right\},
\end{gathered}
$$

with $a / h=2 \pi /(\lambda \sqrt{ } 3)$; see [17].

- See [14], pp. 201-202. 
Let us summarize a few features which stand out in this example:

(a) The scale of the pattern is dependent upon the thickness of the liquid layer, a quantity which can be chosen arbitrarily by the experimenter. Thus we have an example of an "accidental" boundary condition. At the same time we can say that the convection mechanism transmits order: a dimension given by outside conditions is reshaped into a dimension characteristic for the pattern.

(b) The principle of the most unstable solution, which we have invoked, is connected with the circumstance that convection currents initially will arise in a random way, depending upon a chance distribution of spots of high temperature. Once motion has started, viscosity and heat conduction influence its development. The random starting pattern may be considered as a superposition of more or less regular patterns of the kind to which the differential equation refers. For a given mean temperature gradient some of these patterns will be damped; others will show increasing velocities, and it is concluded that the pattern with the greatest rate of increase ultimately will become preponderant. There is consequently a kind of selecting mechanism, which operates on random initial conditions and ultimately favors a definite state of motion. Hence, although the convection mechanism imposes a scale related to the thickness of the liquid layer, we also perceive an influence from initial conditions. We shall later consider an example where the initial conditions are preponderant.

(c) The convection pattern can persist so long as there is a source of energy which supplies the heat, and a sink of energy where the heat is absorbed and radiated or conducted away. Hence the appearance of the pattern needs the presence of another form of order: the existence of different temperatures at the limiting surfaces of the field, while the colder surface must not get saturated but must be able to loose its energy to unlimited surroundings. ${ }^{10}$

${ }^{10}$ The theory of the instability of a layer of viscous material heated from below has been applied by Vening Meinesz to the mantle of the Earth, that is a layer of about $2900 \mathrm{~km}$ thickness between the outer crust of the Earth and the core. This mantle is heated from the inside by radioactive processes in the interior of the Earth. Vening Meinesz has developed a theory according to which instability in this layer is gradually built up in the course of many millions of years; it is released when a certain degree of instability is reached, after which the material in the mantle performs a half turn, which brings it into a stable situation. Since the heating continues, the process repeats itself. It is supposed that it occurs intermittently, as the stresses must exceed a certain threshold before flow can start (the behavior of the material of the mantle is not governed by linear equations). The period might be about 200 million years, which is the period assumed for the major phases of mountain building in the geologic history of the Earth; the mountain building would be a consequence 
5. Longitudinal vortices in shearing flow. Several variations of the convection problem have been considered in the literature. An interesting case is that where the heated liquid is not at rest originally, but has already a shearing motion. This can be produced by giving a horizontal motion to the upper confining surface with respect to the lower surface which supports the liquid. It has been found that in this case the polygonal cell pattern can make place for a system of longitudinal cells, stretched out in the direction of the shearing motion. ${ }^{11}$ These cells are separated from each other by parallel vertical walls, alternately with rising and with descending motion. Each longitudinal cell forms a vortex tube with the axis parallel to the direction of the general flow. When we neglect viscosity and heat conduction and consider the accelerated motion only, a relatively simple differential equation suffices to describe the characteristic features of the pattern. ${ }^{12}$

A related system of vortices has been obtained in the space between two rotating co-axial cylinders, for a liquid not subjected to any gradient of temperature. In this case the instability is produced by the centrifugal forces acting on the liquid, provided a certain relation is satisfied by the angular velocities of the cylinders, dependent upon the radii and upon the viscosity of the liquid. When the width of

of the stresses exerted on the crust when there is flow in the mantle. Vening Meinesz has also worked out the idea that the distribution of the land masses of the Earth may be related to the pattern of convection currents which could have appeared in an earlier part of the Earth's history. This pattern shows a marked preference for spherical harmonic functions of low order, and calculation shows that these low order functions represent the "most unstable" types of motion. A pattern of this type could make understandable why most of the land mass is concentrated in a few large continents, instead of being completely split up into islands of much smaller dimensions. See $[18]$.

1 See [13].

12 When it is assumed that the pattern of motion is independent of $x$ (the coordinate in the direction of the shear flow), the differential equation for the now time dependent vertical velocity $w$ still has the form given in footnote 7 , independently of the velocity profile $U(z)$ of the shear flow. When $\nu$ and $\kappa$ are replaced by zero in this equation, it reduces to:

$$
\sigma^{2} \Delta w=g \alpha \beta \Delta^{*} w,
$$

which, in consequence of $\partial / \partial x=0$, can be written:

$$
\frac{\partial^{2} w}{\partial z^{2}}-\left(\frac{g \alpha \beta}{\sigma^{2}}-1\right) \frac{\partial^{2} w}{\partial y^{2}}=0 .
$$

It can have solutions of the type mentioned in the text if

$$
g \alpha \beta / \sigma^{2}>1 \text {, }
$$

so that $\partial^{2} w / \partial z^{2}$ and $\partial^{2} w / \partial y^{2}$ must have the same sign and thus both can be negative. 
the space between the cylinders is small in comparison with the radii, the equation governing the stability or instability of the motion is related to that which holds for the case of a liquid between flat plates in shearing motion, subjected to a destabilizing temperature gradient. ${ }^{13}$

It is a curious result of observations made by many experimenters that similar longitudinal vortices often appear in boundary layer flow along a flat plate, when there is no temperature gradient at all and when curvature effects as are found between rotating cylinders are entirely absent.

Much mathematical work and thought has been devoted to the problem of the instability of shear flow in such a case. The equation describing the behavior of a perturbation of this flow has a character different from that which presents itself in the problem of the layer heated from below or in that of the flow between rotating cylinders. In particular the role of the viscous friction is quite different and the evaluation of the criterion for instability involves mathematical relations of great complexity. Moreover, when instability occurs, it is found that the solution of most critical instability has vortex motion with the axes of the vortices transverse to the general direction of the flow, so that the disturbed motion is two-dimensional. ${ }^{14}$

13 The original investigations, both theoretical and experimental, have been presented in an important memoir by G. I. Taylor, see [19].

The relation of Taylor's problem to that of the longitudinal vortices produced by temperature instability in shear flow was pointed out by A. R. Low and D. Brunt $[20 ; 21]$. See also [14], pp. 202-206.

Let the radii of the two cylinders be $R_{\theta}$ (outer) and $R_{i}$ (inner); suppose that the outer cylinder is at rest, while the inner one rotates, the undisturbed rotational velocity of the liquid being $U=C\left(R_{e}^{2} / r-r\right)$, where $C$ is a constant. The equation for the radial component $w$ of the perturbation velocity, in the case that viscosity is neglected and that $\left(R_{0}-R_{i}\right) \ll R_{i}$, takes the form:

$$
\frac{\partial^{2} w}{\partial r^{2}}-\left\{\frac{4 C^{2}}{\sigma^{2}}\left(\frac{R_{e}^{2}}{r^{2}}-1\right)-1\right\} \frac{\partial^{2} w}{\partial y^{2}}=0
$$

the coordinate $y$ here being measured in the axial direction. This equation is somewhat more complicated than the equation mentioned in footnote 12 , but nevertheless is related to it.

Görtler has developed a theory for boundary layer flow along curved walls, where again a related form of instability is found. See [22].

${ }^{14}$ Summaries of the theory of the stability of the laminar motion between two flat walls, or in the boundary layer along a flat plate, are given in many textbooks on hydrodynamics. The stability for three-dimensional disturbances was investigated by H. B. Squire [23]. Squire demonstrated "that, if any velocity profile is unstable (against such disturbances) for a particular value of Reynolds' number, it will be unstable at a lower value of Reynolds' number for two-dimensional disturbances." 
It has not been possible thus far to find out definitely why longitudinal vortex motion nonetheless so often makes its appearance. A probable explanation is that as soon as a system of transverse vortices has been formed, the field has become unstable for localized disturbances. When a vortex line extending in the transverse direction has a local bend, the field of induced velocities will make this bend rotate around the line. This will bring the bend into a layer of different horizontal velocity; hence a distortion will set in, in which longitudinal vortex elements are produced. There is experimental evidence for such a process. ${ }^{15}$ But the presence of such distortions does not explain why in fully developed turbulence the longitudinal vortices seem to play a more important part than transverse vortices. It is as if they completely take over the role of the latter in keeping up the mixing process between the horizontal layers, which is characteristic for turbulence. ${ }^{16}$ In their structure and arrangement one can find some analogy with that of the longitudinal vortices obtained as a consequence of temperature instability, or of instability through curvature and centrifugal forces. This might induce us to consider whether a system of equations could be devised that should bring this analogy into evidence.

The forces arising from the presence of turbulence produce both a dissipation and a coupling between the velocity components. When one attempts to linearize the equations and to introduce forces directly depending upon the velocity components (instead of depending upon spatial derivatives), a possible assumption might be:

$$
f_{x}=-k u+K w ; \quad f_{y}=-k v ; \quad f_{z}=-k w-K u ;
$$

where $k$ and $K$ are two coefficients, $k$ determining dissipation and $K$ introducing a coupling between $u$ and $w$. One may expect that the coefficient $K$ will depend upon the derivative $d U / d z$, characteristic for the shearing motion. The desired analogy can be obtained when one treats $k$ as a constant and assumes that $K=\alpha d U / d z$, with $0<\alpha<1$. Although this is no more than a guess, the resulting equations may be of some interest in a discussion of the structure of a turbulent boundary layer. ${ }^{17}$

${ }^{15}$ See F. R. Hama [24; further publications of Professor Hama and his group on this subject are forthcoming]; and J. R. Weske [25].

10 Compare the discussion on the relation between the "momentum transfer theory" and the "vorticity transfer theory" in S. Goldstein [26] and for more details: A. A. Townsend $[27 ; 28 ; 29]$.

17 When these hypothetic forces are introduced into the equations of motion for an incompressible fluid, and when the motion is supposed to be independent of $x$ and viscosity is neglected, the perturbation equations take the form: 
6. Selecting processes in cases where the initial state of the field determines the resulting pattern. In the preceding example we have seen that instability may bring with it a selecting mechanism which can impose a certain pattern upon the resulting field. We now pass on to a third group of cases, in which the resulting pattern is wholly determined.by features present in the initial state of the field, in particular, by features that appear to have the greatest speed of propagation.

The simplest example is a collection of freely moving, noninteracting and noncolliding particles, with velocities of all possible magnitudes and directions, the particles initially being distributed over a finite volume surrounded by empty space. When such a system is left to itself, a spherically symmetric field of divergent motion will gradually arise, with rough proportionality between speed and distance from the center of the original location. The example looks trivial, but it is a stepping stone toward more complicated phenomena. ${ }^{18}$ The

$$
\begin{aligned}
\frac{\partial u}{\partial t}+w \frac{d U}{d z} & =-k u+K w \\
\frac{\partial v}{\partial t} & =-\frac{1}{\rho} \frac{\partial p}{\partial y}-k v \\
\frac{\partial w}{\partial t} & =-\frac{1}{\rho} \frac{\partial p}{\partial z}-k w-K u ; \\
\frac{\partial v}{\partial y}+\frac{\partial w}{\partial z} & =0 .
\end{aligned}
$$

Elimination of the pressure leads to (treating $k$ as a constant):

$$
\begin{aligned}
& \left(\frac{\partial}{\partial t}+k\right) u=-\left(\frac{d U}{d z}-K\right) w \\
& \left(\frac{\partial}{\partial t}+k\right) \Delta w=-K \frac{\partial^{2} u}{\partial y^{2}} .
\end{aligned}
$$

We write $\sigma$ for $\partial / \partial t+k$, and eliminate $u$. The resulting equation can be written:

$$
\frac{\partial^{2} w}{\partial z^{2}}-\left\{\frac{K(d U / d z-K)}{\sigma^{2}}-1\right\} \frac{\partial^{2} w}{\partial y^{2}}=0
$$

This is again an equation of the same type as considered in footnote 12 , provided that $K(d U / d z-K)$ has a positive value.

The subject has been considered by the present author in [30].

$18 \mathrm{~A}$ set of particles, moving with all possible speeds in all possible directions, has been used by Milne as the starting point for a theory of relativity and gravitation. See: E. A. Milne [31].

A case related to that considered in the text is obtained with surface wave motion on deep water. Suppose that a limited area of a large ocean is acted upon by a random fluctuating pressure field. The pressure will produce displacements of the surface of the water, which can be analyzed into a complex of wave systems with different wave- 
ordering which appears in this case is not in contradiction with the principles of thermodynamics: the assumption that the system of particles originally is found in a finite volume amidst emptiness represents an order in location, and during the motion of the system this order in location is transformed into a relation between velocity and location. When the system contains a sufficiently large number of particles, a distribution function for the velocity components $\xi_{h}$ can be introduced, as is used in the kinetic theory of gases. This distribution function is subjected to the equation:

$$
\frac{\partial F}{\partial t}+\xi_{h} \frac{\partial F}{\partial x_{h}}=0 .
$$

There is no collision term on the right hand side, and calculation shows that the integral which determines the entropy of the arrangement, does not change in time. ${ }^{19}$

In treatises on the kinetic theory of gases it is shown that, by calculating the moments of the Boltzmann equation for the distribution function (of which the equation given above is a simplified example), equations can be deduced for the change of the mean local density and of the mean local flow velocity in the course of time. In the case considered above the equations obtained are those for a gas in which there would be no collisions and in which the notion of pressure is becoming vague. The scale of the resulting pattern of motion is exclusively determined by what happens to be given in the initial state.

A more realistic case is obtained when interactions between the particles are introduced, that is, when we admit collisions. The pattern of motion which now develops will depend upon the propagation of waves through the system. The wave speed is dependent upon the speed of the gas itself; this has the consequence that waves carrying motion of the gas in the direction of their propagation will become steeper and finally develop an abrupt front, called a shock front from the sudden way in which it imparts motion to the gas upon which it impinges.

lengths. The waves, once produced, propagate themselves in directions normal to their crest lines. Since the waves with longer wavelengths have a larger velocity of propagation than shorter waves, it is found that at a certain distance from the region which had been disturbed by the pressure fluctuations, a more or less regular pattern of long waves makes its appearance. In virtue of their greater speed these waves have run ahead of the shorter waves. Thus there develops a definite pattern, not present in the original disturbance.

${ }^{10}$ The integral

$$
H=-\iint d x d \xi F \ln F,
$$

extended over all space and over all possible velocities, is independent of the time. 
A shock front is a, usually very narrow, transition zone in which collisions impart motion to the molecules reached by the wave. It can have a complicated structure, dependent upon the interactions brought about by the collisions. These interactions are of various kinds. In the first place there is exchange of translational motion; this exchange occurs in a few collisions and determines the change of mean velocity and the primary change of density. When the molecules are diatomic, there is also exchange of rotational energy, which takes place almost as rapidly. There can further be exchange of vibrational energy; this is a process which often needs hundreds or many thoussands of collisions before it becomes effective. Other features can be electronic excitation, ionization, dissociation or chemical reactions, and radiation. Complicated interactions occur when there is a magnetic field; the shock front may then become a current sheet, carrying an electric current in a direction perpendicular to that of the motion of the wave front. All these processes have their peculiar rates of growth and the consequence is that behind, and in certain cases also ahead of the front at which the change of mean velocity occurs, there can extend regions in which various adjustments take place.

In this example we find evidence for two types of structure or, as we may say, two types of order. One refers to the structure of the shock wave; the other results as a pattern formed by the propagation of many shock fronts through space. From the molecular point of view, the structure of the shock wave is a statistical phenomenon expressing itself in the distribution of number densities and of states of energy, and regulated by the various processes occurring in collisions between the molecules. On the other hand, the pattern formed by the location of several shock fronts in space at a given instant of time is dependent upon what was given in the initial state, so that it has an accidental character. The question may turn up whether some features of these patterns can have similar forms for a variety of initial states. This brings us to the concept of random initial data, and we are led to consider the question: are there aspects of statistical order in the patterns which arise from such random data? In the next section I will discuss a particular example.

7. Example of a pattern arising from random initial data. We consider the partial differential equation $:^{20}$

$$
\frac{\partial u}{\partial t}+u \frac{\partial u}{\partial x}=\nu \frac{\partial^{2} u}{\partial x^{2}} .
$$

${ }^{20}$ See [32]. 
This equation has a form which is related to that for one-dimensional motion in gas dynamics for a gas under such circumstances that there would be no pressure (this would require zero temperature, and immediate loss by radiation of all heat produced by compression). Positive values of $t$ are considered. The initial values of the function $u(x, t)$ will be written $u_{0}(x)$, defined for $-\infty<x<+\infty$. The parameter $\nu$ is positive and very small. We look for the form which the solutions assume when $\nu \rightarrow 0$.

The equation can be reduced to a linear one when we write: ${ }^{21}$

$$
u=-2 \nu \partial(\ln w) / \partial x .
$$

The auxiliary variable $w$ must satisfy the equation:

$$
\frac{\partial w}{\partial t}=\nu \frac{\partial^{2} w}{\partial x^{2}} \text {. }
$$

The solution of the latter equation, determined by the initial condition prescribed for $u$, can be written in the form:

$$
w=\frac{1}{2(\pi \nu t)^{1 / 2}} \int_{-\infty}^{+\infty} d \xi \quad \exp \left\{\frac{-(\xi-x)^{2}}{4 \nu t}-\frac{1}{2 \nu} \int_{0}^{\xi} u_{0}\left(\xi^{\prime}\right) d \xi^{\prime}\right\} .
$$

When $\nu$ goes to zero, the solution for $u$ can be obtained by means of a geometrical construction. In an auxiliary diagram with horizontal coordinate $\xi$ we construct a curve determined by:

$$
z(\xi)=-\int_{0}^{\xi} u_{0}\left(\xi^{\prime}\right) d \xi^{\prime}
$$

We suppose that $u_{0}(x)$ is a fluctuating function with positive and negative values in equal probability; in that case the integral curve $z(\xi)$ also will represent a fluctuating function. Next we introduce a parabola $\mathcal{Z}(\xi)=(\xi-x)^{2} / 2 t+C$ ( $C$ being a constant), where $x$ and $t$ refer to the point and the instant for which we wish to know the value of $u$. When $C$ is positive and very large, the parabola will be situated far above the curve $z(\xi)$. We gradually reduce $C$, until the parabola touches the curve $z(\xi)$ from above for the first time. The point of contact $\xi_{m}$ then fixes the value of $u(x, t)$ through the relations:

$$
z=\mathrm{Z} ; \quad d z / d \xi=d \mathrm{Z} / d \xi
$$

leading to

$$
u(x, t)=u_{0}\left(\xi_{m}\right)=\left(x-\xi_{m}\right) / t,
$$

${ }^{21}$ The reduction was given by E. Hopf and J. D. Cole. See $[33 ; 34]$. 
so that:

$$
u(x, t)=u_{0}(x-u t) .
$$

All interesting features of the problem are contained in this procedure. One can imagine that it is carried out by a machine, which makes the parabola glide over the $z$-curve and which reads off the points of contact and the directions of the common tangents to the parabola and the z-curve. Time and again there will be cases where the parabola touches the z-curve in two points simultaneously, so that the contact point suddenly jumps from one position to another position, at a finite distance from the first one. This means that there is a sudden change in the value of $u(x, t)$; thus the solution of the equation presents "discontinuities." The jumps are always downward $:^{22}$ on the right hand side of a jump $u$ has a value below the one it had on the left hand side. (Actually the jumps are not mathematical discontinuities: $\partial u / \partial x$ assumes negative values of the order $1 / \nu$.)

The fact that such jumps appear in the solution shows that out of the randomness of the initial data the mechanism represented by the differential equation produces something which has greater specificity than was apparent at first sight. There is an analogy with the appearance of shock waves in a gas, of which this mathematical example gives a simplified version.

The procedure for solving the equation brings a mechanical separation between the initial data, which are embodied in the $z$-curve; and the operation of the differential equation, represented by the gliding parabola, which can be machined according to the value of $t$ to be considered. Consequently the method is eminently suitable for the investigation of features which may appear when a random collection of initial data is considered. We have only to prepare a random set of z-curves and to study various statistical aspects of the behavior of the parabola gliding over them. The randomness of the initial data may be limited by some condition of general nature; for instance, there may be a limitation for $\left|u_{0}(x)\right|$. More important is to require the existence of a finite and integrable correlation function $R(a)=$ $\left\langle u_{0}(x) u_{0}(x+a)\right\rangle$, where $a$ can go from zero to infinity, while the value of $R(a)$ must be independent of $x$.

Statistical features of great interest are the distribution function governing the widths of the jumps and the statistical relations involved in the position of the two contact points on a parabola with respect to the axis of the parabola. It can be shown that all the

${ }^{22}$ Upward jumps would occur when the plus sign before the second term on the left hand side of the equation is replaced by a minus sign. 
correlative properties of the function $u(x, t)$ with reference to $x$ for a given $t$, can be calculated from these statistical data.

When $t$ increases more and more, an asymptotic theory of the statistics of the jumps can be worked out. For this purpose the z-curve is considered as picturing the vertical motion of a particle subjected to random up and down disturbances, while at the same time the particle is transported in the horizontal direction with constant unit velocity. A random collection of initial data can then be considered as the picture of a set of particles, subjected to a process of "diffusion" in the vertical direction. It is found that the coefficient of diffusion is ultimately determined by the integral of the correlation function for the initial data:

$$
J=\int_{0}^{\infty} d a\left\langle u_{0}(x) u_{0}(x+a)\right\rangle .
$$

The statistics of double contacts can then be obtained from a calculation of the chance that a particle, whose path comes close to the parabola at a point $\xi_{1}$, reaches the parabola again at a point $\xi_{2}$, while the path remains below the parabola everywhere else (also before it reaches $\xi_{1}$, and after it has passed $\xi_{2}$ ).

The calculation of this chance requires that certain special solutions are constructed of the diffusion equation for a domain bounded by a parabolic curve. The difficulties involved in defining the measure associated with the notion of probability can be evaded for a large part by considering an asymptotic case referring to very large values of the time; this is a requirement that occurs on several occasions in the treatment of the problem. It then appears to be possible to work out the probability relations and to write down a set of expressions describing the asymptotic statistics of the jumps. The expressions require only quadratures, but each of them contains a large series of integrations and the integrands depend upon solutions of the diffusion equation. All expressions are completely definite; the only parameters which remain in the results are the quantity $J$ defined above, and the time $t$; there are no coefficients which are not fully determined by the integrals. ${ }^{28}$

${ }^{23}$ In the asymptotic case the auxiliary diffusion problem is governed by the equation

$$
\frac{\partial \psi}{\partial \xi}=J \frac{\partial^{2} \psi}{\partial z^{2}},
$$

where $\psi$ measures the density of the diffusing particles in a small element of the $\xi$, $z$-plane. In the asymptotic case, moreover, we may consider the motions before the point $\xi_{1}$ is reached, those between $\xi_{1}$ and $\xi_{2}$, and those beyond the point $\xi_{2}$ as prac- 
8. Concluding remarks. We have gone, rather rapidly, through a few examples exhibiting the appearance of forms of order. In each case we have looked for the features which were responsible for the order.

The most pronounced forms of order are directly related to the fundamental laws of nature governing the behavior of electrons, protons and other elementary particles involved in the structure of atoms and molecules. In other problems of mathematical physics the pattern was dependent, sometimes partially and sometimes completely, upon boundary conditions or initial conditions. In comparison with the fundamental laws we have used the term "accidental circumstances" for these conditions. However, the "accidental circumstances" are the outcome of previous happenings; in other words,

tically independent of each other. This has the consequence that the probability of making contact with the parabola at the points $\xi_{1}$ and $\xi_{2}$, while remaining below the parabola everywhere else, can be expressed as a product of three functions:

$$
\text { const. } E\left(-\xi_{1}\right) \cdot \Psi\left(\xi_{1}, \xi_{2}\right) \cdot E\left(\xi_{2}\right) d L d B \text {, }
$$

with $L=\xi_{2}-\xi_{1} ; H=Z_{2}-Z_{1}=\left(\xi_{2}^{2}-\xi_{1}{ }^{2}\right) / 2 t$.

In order to define the functions appearing in this formula we consider a solution $\psi\left(\xi_{2}, z_{2} ; \xi_{1}, z_{1}\right)$ of equation $\left({ }^{*}\right)$ which gives the density of diffusing particles at the point $\xi_{2}, z_{2}$, when there is a unit source at $\xi_{1}, z_{1}$, under the conditions:

$$
\Delta_{1}=\xi_{1}^{2} / 2 t-z_{1}>0 ; \quad \xi_{2}>\xi_{1} ;
$$

(the latter two conditions ensure that both the source point and the point of observation are situated below the parabola); and finally:

$$
\psi=0 \text { on the parabola } \xi^{2} / 2 t-z=0 .
$$

We then reduce the values of $\Delta_{1}$ and $\Delta_{2}$, and introduce the limit:

$$
\Psi\left(\xi_{1}, \xi_{2}\right)=\lim _{\Delta_{1}=0, \Delta_{2}=0} \frac{\psi\left(\xi_{2}, z_{2} ; \xi_{1}, z_{1}\right)}{\Delta_{1} \Delta_{2}} .
$$

Next we consider a solution $\psi\left(\xi, z ; \xi_{2}, z_{2}\right)$, with the unit source at $\xi_{2}, z_{2}$, while $\xi$, $z$ is the observation point, with $\xi>\xi_{2} ; \xi^{2} / 2 t-z>0$; and $\Delta_{2}$ as before. We introduce the limit:

$$
E\left(\xi_{2}\right)=\lim _{\Delta_{2}=0, \xi \rightarrow \infty} \int_{-\infty}^{\xi_{2} / 2 t} d z \frac{\psi\left(\xi, z ; \xi_{2}, z_{2}\right)}{\Delta_{2}} .
$$

The function $E\left(-\xi_{1}\right)$ can be defined by means of a diffusion process with a flow of unit velocity going in the negative $\xi$-direction; the result is that $E\left(-\xi_{1}\right)$ is the same function as the one defined by the limit above, with $-\xi_{1}$ substituting for $\xi_{2}$.

Expressions for the correlation functions associated with the asymptotic form of the solution of the partial differential equation given at the beginning of section 7 of the text, have been developed in [35]; their connection with integrals depending upon the functions $\psi$ and $E$ has been considered in [32], pp. 417-421. The integrals are of a complicated nature. In order to work them out the solution of the diffusion equation with $\psi=0$ on a parabolic boundary must be obtained. 
they are dependent upon previous forms of order. In this sense we have already spoken of a transmission of order. We have also pointed out that in this scheme of thought the large-scale nonuniformities in the universe must be counted as forms of order; this refers, for instance, to the presence of concentrated matter and of concentrated sources of energy amidst almost empty space. It is well known that in all theories about the origin of the solar system or the origin of galaxies assumptions turn up concerning the effects of such nonuniformities.

Hence every explanation of order must refer to "given things," whether these be distribution of matter, or the nature of space and time, or quantum physical laws.

Transmission of order is, of course, nothing else than the network of causal relationship. From quantum theory it follows that this relationship is imperfect when we attempt to describe phenomena of atomic dimensions in the way in which one is accustomed to do this with large scale phenomena. Inseparably connected with this result is the fact that no physical observation can be carried through without disturbing the system upon which the observation is made and that the most complete observation (the so-called "maximum observation") can give us no more than half of the data which we would need in a classical description of motions taking place in space and time. The consequence is that each situation, in so far as it can be known to us through an observation, will give rise, in its development, to the appearance of a complex of competing configurations instead of leading to a single completely determined result. In broad terms this is the consequence of the existence of a great number of different quantum states corresponding to a single energy level for the system plus those surroundings which are needed in the observation. The multiplicity of states can be reduced when we can bring the system to a level of lower energy, by radiation of energy into empty space or by other means for detracting energy from it. This is the way in which order is produced in an important number of forms in nonliving nature, and it is conditioned by the existence in our universe of large domains which are practically devoid of matter and energy.

We also found that forms of order can arise in patterns of flow, when there is a constant transport of energy through the system, which keeps the system in motion against the damping influences of viscous friction, or of heat conduction, and in other cases, of diffusion. Here again the order is a by-product, so to say, from a more embracing process in which order is dissipated.

Thus in connection with all cases of emergence of order we found 
accompanying forms of loss of order. This has sometimes raised the question whether the universe is only "running down," or whether there could be creation of new order.

In considering the problem which presents itself here, we must keep in mind that the distinction between order and disorder is a distinction outside the subject matter of physics. Physical nature is a whole and no configuration counts for more in it than any other configuration. The analytic point of view which studies forms of order and compares them and classifies them, is an outside point of view. When we say that crystallization produces order, at the expense of disorder in the liquid out of which the crystals have come forward, it is our distinction which fixes attention upon the crystals and treats them as something that has more meaning than the liquid. When we speak of a statistical order, it is we who are interested in certain mean values or in recurrent events, and who count them and describe them by means of some formula. In any description of nature, in any formulation of a physical situation, we bring in subjective features. We see and act upon what is around us only through the effect of operations which introduce demarcations, that is, which give local accents and introduce spotlights. Our biological structure, of which our spiritual structure is an outcome, apparently drives us to make distinctions. Without this we would be no more than another physical process and there would be no understanding and no science. This feature of making distinctions, of having conceptions, is something which is not itself embodied in the description of physics. It makes possible logical and mathematical analysis and permits us to conceive alternative situations. It may stand in relation to physics as a "metaphysics," in a somewhat similar sense as the term "metamathematics" is used in the analysis of the structure of mathematics.

I believe that this power of accentuating, this power of selection is a feature which pervades all phenomena of life. It must derive from something which has a meaning in the whole of nature. I believe that there is some form of coordination in the universe which is left out when we construct our descriptions according to the current methods of mathematics and physics. This omission has proved its good right and usefulness in physical theory, but perhaps it may not be applicable to all that happens. It may be that the bookkeeping of statistical mechanics, in which every configuration is counted as having the same weight, is too crude for a deeper form of understanding and cannot lead to the right answers in all cases. The cases where it does not hold completely might be those where we say: here is a feature of life; here we perceive that we have not a physical system, but a 
living organism before us.

In our minds this particular feature gives us the feeling of being an experiencing subject, integrating its experiences with valuations. It leads us to make distinctions between situations which are not based exclusively on causal relationship with past situations, but are conceived in view of possibilities for a future tempting us to make selections. The diversity of configurations will then not be just a number, only to be counted in the calculation of entropy, but presents itself as a meaningful diversity, offering a possibility of richness in anticipated experience. The idea of perceiving a purpose might be a reflection of this feature.

The living world presents many aspects which, when taken in their entirety, go far beyond what we know from nonliving things, notwithstanding obvious and extensive forms of analogy observed when isolated reactions in living organisms are compared with reactions known from nonliving nature. With a few species of atoms, there is a far greater diversity in molecular structures than is found in all the minerals. We find composite organs, in which ordered parts are combined hierarchically as elements in highly elaborate arrangements, reaching in complexity and coordination far beyond all that we know from inorganic systems (here I mean, of course, systems not built by man, for systems built by man are part of life's activity). We find systems of complicated responses to stimuli coming from outside. Moreover, there is a stability in the transmission of order, which transmission has taken place uninterruptedly through more than a billion years of geologic history, with a precision compared to which the transmission of order in the lattice structure of even the largest single crystal known in nature is almost futile. Finally, out of life have arisen the notions of individuality, of consciousness and personality, of aesthetic and of moral order, and of generosity. We have found a sense in order - a conception extraneous to mathematical and physical relations.

I would believe that these features are the outcome of something acting in the whole universe. It must be as fundamental as the aspects which are studied in physics and mathematics. It would be something which embodies an appreciation for contrasts. Apparently it has found its widest scope on Earth in the possibilities afforded by what we call the "organic compounds," but it might be that we owe to its grace also something of the beauty of nonliving things, as crystals. Extending the usual meaning of the word, I would call biology the study of this coordinating activity in all its forms.

Thus in the living world the subject of order has extra dimensions, 
dimensions which we leave aside in the picture of physics, although we make use of them when we bring this picture into words and equations.

It will be recognized that the ideas which I have discussed in these last remarks, have been derived from the writings of Alfred North Whitehead. ${ }^{24}$ I have taken the liberty to bring them in as a kind of counterpart to the statistical picture which is paramount in physics, and which has been an important part of the work of Josiah Willard Gibbs.

In my main talk I have restricted myself to forms of order which we find in nonliving nature, as was proper. But I would not omit to hint at the possibility of a wider point of view, in which the notion of order must find a more complete realization. For it is only in the realm of conceptual activity that we can analyze order and present it in a mathematical form, and what is not less important, that we can find joy and inspiration in relations which we have understood.

\section{BiBLIOGRAPHY}

1. W. Shockley and others, Imperfections in nearly perfect crystals, Wiley, New York, 1952.

2. H. G. van Bueren, Imperfections in crystals, North-Holland Publ. Co., Amsterdam and New York, 1960.

3. W. T. Read, Dislocations in crystals, McGraw-Hill, New York, 1953.

4. J. M. Burgers and W. G. Burgers, Dislocations in crystal lattices, Chapter 6 of Rheology. Theory and applications, edited by F. R. Eirich, Vol. I, pp. 141-199, Academic Press, New York, 1956.

5. A. Verma, Crystal growth and dislocations, Butterworth, London, 1953.

6. W. Dekeyser et S. Amelinckx, Les dislocations et la croissance des cristaux, Masson, Paris, 1955.

7. M. L. Kronberg, Plastic deformation of single crystals of sapphire: Basal slip and twinning, Acta Metallurgica 5 (1957), 507-524.

8. S. Amelinckx, W. Bontinck, W. Dekeyser and F. Seitz, On the formation and properties of helical dislocations, Philos. Mag. 2 (1957), 355-378.

9. W. Bontinck, Climb phenomena in synthetic fluorite crystals, Philos. Mag. 2 (1957), 561-567.

10. W. C. Dash, Generation of prismatic dislocation loops in silicon crystals, Phys. Rev. Lett. 1 (1958), 400-402.

11. Henri Bénard, Les tourbillons cellulaires dans une nappe liquide transportant de la chaleur en régime permanent, Annales de Chimie et de Physique (7e série) 23 (1901), 62-144.

12. - Les tourbillons cellulaires dans une nappe liquide, Revue Générale des Sciences 11 (1900), 1261-1271, 1309-1328.

13. K. Chandra, Instability of fluids heated from below, Proc. Roy. Soc. London. Ser. A 164 (1938), 231-242.

${ }^{24}$ The ideas expressed in the text are contained in the philosophical works of A. N. Whitehead, see $[36 ; 37 ; 38]$. (Added November 1962: I hope to present elsewhere a more extensive discussion of these ideas.) 
14. H. Jeffreys, Some cases of instability in fluid motion, Proc. Roy. Soc. London. Ser. A 118 (1928), 195-208.

15. W. H. Reid and D. L. Harris, Some further results on the Benard problem, Phys. Fluids 1 (1958), 102-110.

16. J. Wasiutynski, Studies in hydrodynamics and structure of stars and planets, Astrophys. Norvegica 4 (1946), pp. 153-165.

17. D. C. Christopherson, Note on the vibration of membranes, Quart. J. Math. 11 (1940), 63-65.

18. F. A. Vening Meinesz, A remarkable feature of the earth's topography; origin of continents and oceans, Proc. Roy. Netherl. Acad. Sciences, Ser. B 54 (1951), 212-228; Convection currents in the earth and the origin of the continents, ibid. 55 (1952), 527-553.

19. G. I. Taylor, Stability of a viscous liquid contained between two rotating cylinders, Philos. Trans. Roy. Soc. London, Ser. A 223 (1923), 289-343.

20. A. R. Low and D. Brunt, Instability of viscous fluid motion, Nature 115 (1925), 299-301.

21. A. R. Low, On the criterion for stability of a layer of viscous fluid heated from below, Proc. Roy. Soc. London Ser. A 125 (1929), 180-195.

22. H. Görtler, Ueber eine dreidimensionale Instabilität laminarer Grenzschichten an konkaven Wänden, Göttinger Gesellsch. d. Wissenschaften, Nachr. a.d. Mathematik 2. No. 1 (1940).

23. H. B. Squire, On the stability for three-dimensional disturbances of viscous fuid flow between parallel walls, Proc. Roy. Soc. London, Ser. A 142 (1933), 621-628.

24. F. R. Hama, J. D. Long and J. C. Hogarthy, On transition from laminar to turbulent flow, Technical Note BN-81, Institute for Fluid Dynamics and Applied Mathematics, University of Maryland, August, 1956.

25. J. R. Weske, Experimental study of detail phenomena of transition in boundary layers, Technical Note BN-91, ibidem, February, 1957.

26. S. Goldstein, Modern developments in fluid dynamics, Oxford Univ. Press, Oxford, Vol. I, 1938, pp. 210-214.

27. A. A. Townsend, The eddy viscosity in turbulent shear flow, Philos. Mag. 41 (1950), 890-906.

28. - The structure of the turbulent boundary layer, Proc. Cambridge Philos. Soc. 47, (1950), 375-395.

29. - The structure of turbulent shear flow, Cambridge Univ. Press, Cambridge, 1956, p. 114.

30. J. M. Burgers, Some considerations on turbulent flow with shear, Proc. Roy. Netherl. Acad. Sciences, Ser. B 56 (1953), 125-147.

31. E. A. Milne, Relativity, gravitation and world structure, Oxford Univ. Press, Oxford, 1935.

32. J. M. Burgers, Further statistical problems connected with the solution of a simple nonlinear partial differential equation, Proc. Roy. Netherl. Acad. Sciences, Ser. B 57 (1954), 159-169; 403-433.

33. E. Hopf, The partial differential equation $u_{t}+u u_{x}=u_{x x}$, Comm. Pure Appl. Math. 3 (1950), 201-230, in particular p. 203.

34. J. D. Cole, a private communication (1949).

35. J. M. Burgers, Correlation problems in a one-dimensional model of turbulence, Proc. Roy. Netherl. Acad. Sciences 53 (1950), 247-260; 393-406.

36. A. N. Whitehead, Science and the modern world, Macmillan, New York, 1925. 37. - , Process and reality, Cambridge Univ. Press, Cambridge, 1929.

38. - Adventures of ideas, ibidem 1933.

UNIVERSITY OF MARYLAND 